\title{
Thermo-hyperelastic models for nanostructured materials
}

\author{
WANG ZhiQiao ${ }^{1,2} \&$ ZHAO YaPu $^{1 *}$ \\ ${ }^{1}$ State Key Laboratory of Nonlinear Mechanics (LNM), Institute of Mechanics, Chinese Academy of Sciences, Beijing 100190, China; \\ ${ }^{2}$ School of Engineering and Technology, China University of Geosciences, Beijing 100083, China
}

Received December 10, 2010; accepted February 25, 2011; published online March 14, 2011

\begin{abstract}
In the framework of continuum thermodynamics, the present paper presents the thermo-hyperelastic models for both the surface and the bulk of nanostructured materials, in which the residual stresses are taken into account. Due to the existence of residual stresses, different configuration descriptions of the surface (or the bulk) thermo-hyperelastic constitutive equations are not the same even in the cases of infinitesimal deformation. As an example, the effective thermal expansion coefficient of spherical nanoparticles is analyzed.
\end{abstract}

nanostructured materials, thermo-hyperelastic, surface, residual stresses

PACS: 65.40.De, 62.25.-g, 68.65.-k

\section{List of main symbols}

1 identity tensor in 3D Euclidean space

$\boldsymbol{A}_{i} \quad$ covariant base vectors of the reference configuration

$\boldsymbol{C}, \boldsymbol{C}^{*}, \hat{\boldsymbol{C}}$ Green deformation tensor defined from the reference to the current configurations, from the stress-free to the reference configurations and from the stress-free to the current configurations, respectively

$C_{s E}, C_{E} \quad$ surface and bulk specific heat at constant volume

$\boldsymbol{E}, \boldsymbol{E}^{*}, \hat{\boldsymbol{E}}$ Green stain tensor defined from the reference to the current configurations, from the stress-free to the reference configurations and from the stress-free to the current configurations, respectively

$\boldsymbol{E}_{s} \quad$ surface Green strain tensor

*Corresponding author (email: yzhao@imech.ac.cn)
$\boldsymbol{F}, \boldsymbol{F}^{*}, \hat{\boldsymbol{F}}$ deformation gradient defined from the reference to the current configurations, from the stress-free to the reference configurations and from the stress-free to the current configurations, respectively

$\boldsymbol{F}_{s}, \boldsymbol{F}_{s}^{-1} \quad$ surface deformation gradient and its inverse

$\boldsymbol{F}_{s}^{(o)}, \boldsymbol{F}_{s}^{-1(o)}$ out-plane terms of $\boldsymbol{F}_{s}$ and $\boldsymbol{F}_{s}^{-1}$

$i \quad$ index ranging over the integers 1,2 and 3

$\boldsymbol{I}_{0} \quad$ identity tensor on the tangent plane of the surface in the reference configurations

$J, J^{*} \quad$ the determinants of $\boldsymbol{F}$ and $\boldsymbol{F}^{*}$, respectively

$J_{1}, J_{2} \quad$ two invariants of the surface right stretch tensor

$k_{s}, k_{b} \quad$ surface and bulk thermal conductivities

$L \quad$ elasticity tensor defined in the reference configuration

$\boldsymbol{L}_{1} \quad$ stress-temperature modulus defined in the reference configuration

$\boldsymbol{P}_{i} \quad$ covariant base vectors of the stress-free configuration

$q_{b}^{0} \quad$ heat flows entering the surface from the bulk 
defined in the reference configurations

$\boldsymbol{q}_{s}^{0}, \boldsymbol{q}_{b}^{0} \quad$ surface and bulk heat fluxes defined in the reference configuration

$\hat{\boldsymbol{q}}_{b}^{R} \quad$ initial value of $\boldsymbol{q}_{b}^{0}$

$r_{0}, \bar{r} \quad$ radii of nanoparticles in the reference and the stress-free configurations

$r_{s}^{0}, r_{b}^{0} \quad$ surface and bulk heat supply defined in the reference configuration

$\boldsymbol{S} \quad$ first kind Piola-Kirchhoff stress

$\boldsymbol{S}_{s} \quad$ first kind Piola-Kirchhoff stress of the surface

$\boldsymbol{T}_{R} \quad$ bulk residual stress in the reference configuration

$\boldsymbol{T}_{s} \quad$ second kind Piola-Kirchhoff stress of the surface

$T_{s}, T_{b} \quad$ surface and bulk temperature, respectively

$T_{s}^{0}, T_{b}^{0} \quad$ surface and bulk reference temperature, respectively

$\boldsymbol{u}$ displacement measured from the reference configuration

$\boldsymbol{U}_{s} \quad$ surface right stretch tensor

$w_{s}^{0} \quad$ surface free energy per unit area defined in the reference configuration

$\alpha_{e} \quad$ effective thermal expansion coefficient of nanoparticles

$\beta_{s}, \beta_{b} \quad$ surface and bulk coefficients related to thermal expansion

$\gamma$ surface energy per unit area defined in the current configuration

$\gamma_{0}^{*}, \gamma_{1}^{*}, \gamma_{1}$ surface tension, surface Lamé constants

$\varepsilon_{s}^{0} \quad$ surface internal energy per unit area defined in the reference configuration

$\eta_{s}^{0} \quad$ surface entropy per unit area defined in the reference configurations

$\eta \quad$ bulk entropy per unit volume

$\eta_{R} \quad$ residual value of $\eta$ in the reference configuration

$\lambda, \mu \quad$ bulk Lamé constants

$\theta_{s}, \theta_{b} \quad$ surface and bulk temperature change, respectively

$\boldsymbol{\sigma}, \boldsymbol{\sigma}_{s} \quad$ Cauchy stress and surface Cauchy stress, respectively

$\nabla_{0}, \nabla_{0 s} \quad$ bulk and surface gradient operators defined in the reference configuration

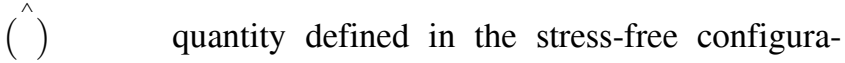
tion

\section{Introduction}

Low-dimensional nanostructured materials, such as nanofilms, nanowires and nanoparticles, have found plenty of applications as nano components of electronic devices, sensors, actuators and nanoelectromechanical systems (NEMS). The mechanical behaviors of aforementioned NEMS devices at the nanoscale, especially nanostructured component failure, are inevitably temperature-related $[1,2]$. Nevertheless, few of the theoretical studies investigated the size effects on the mechanical properties with temperature effect [3-5]. As a matter of fact, for the design and fabrication of reliable NEMS devices, a better understanding is needed for the temperature-dependent mechanical behaviors of such materials [6].

To study the temperature-related mechanical properties of nanomaterials, researchers have developed multiscale computational models through utilizing the Cauchy-Born (CB) hypothesis bridging the molecular descriptions of crystalline configurations and the continuum theories of crystal mechanics [7-9]. Due to very large surface-to-volume ratios, surface effects can significantly affect the macroscopic properties of nanomaterials, which prompted the establishment of models that use a surface and bulk decomposition to study the coupled thermomechanical behaviors of these materials. A temperature-related Cauchy- Born (TCB) rule has been formulated for multiscale modeling the thermomechanical behavior and material stability of crystalline solids in [8,9]. Recently, surface Cauchy-Born (SCB) formulations considering thermal effects have been introduced in [10], which extended the traditional bulk Cauchy-Born theory by augmenting the bulk energy density with a surface energy density that enables it to capture nanoscale surface effects. These SCB models have obvious advantages as they enable the solution of three-dimensional boundary value problems including surface effects utilizing traditional nonlinear finite element methods, and make it computationally tractable, particularly for large systems, that direct atomistic simulations can hardly model.

Theoretically, based on the Gibbs approaches and continuum mechanics, thermodynamic models of the elastic surfaces have been established, e.g., by Murdoch [11], Rusanov [12], Javili and Steinmann [13] and references therein. By combining thermoelastic models for surface and bulk, the size-dependent thermomechanical properties of nanomaterials have been investigated [14-17]. Usually, in the absence of external mechanical or thermal loadings, the surfaces of a nanostructure will be subjected to residual surface stresses, and an elastic field in the bulk materials will be induced by such residual surface stresses from the point of view of equilibrium conditions [18-22]. This self equilibrium state without external loadings is usually chosen as the reference configuration, from which nanostructures will deform. That is to say, both the surface and the 
bulk deform due to the residual stress states. The elastic models of the surface and the bulk with residual stresses for nanostructures have been investigated by the present authors recently [21]. It is pointed out that even in the case of infinitesimal deformation, the referential and current configuration descriptions of surface elasticity should be discriminated due to the existence of surface residual stresses $[19,21]$.

Motivated by the above circumstances, we generalize our work [21] to temperature-dependent cases in this paper, and take thermal effects into account in the thermomechanical analyses of low-dimensional nanostructured materials under the framework of thermodynamics.

\section{Surface thermo-hyperelasticity}

From Gibbs' point of view, surface energy is an excess quantity, which is dependent on not only the surface strain but also the temperature. In this section, we investigate the temperature-related surface elastic model.

\subsection{Thermodynamics of solid surfaces}

Under the action of external loadings or changing the environmental temperature, nanostructured materials will deform from the stressed reference configuration. In the reference configuration, the local forms of solid surfaces energy balance and Clausius-Duhem inequality hold [11]:

$$
\boldsymbol{T}_{s}: \dot{\boldsymbol{E}}_{s}-\nabla_{0 s} \cdot \boldsymbol{q}_{s}^{0}+q_{b}^{0}+r_{s}^{0}=\dot{\varepsilon}_{s}^{0}
$$

and

$$
-\nabla_{0 s} \cdot\left(\frac{\boldsymbol{q}_{s}^{0}}{T_{s}}\right)+\frac{q_{b}^{0}}{T_{b}}+\frac{r_{s}^{0}}{T_{s}} \leqslant \dot{\eta}_{s}^{0},
$$

where $\boldsymbol{T}_{s}$ is work conjugate to $\dot{\boldsymbol{E}}_{s}$, and $\boldsymbol{T}_{s}$ and $\boldsymbol{E}_{s}$ are the second kind surface Piola-Kirchhoff stress tensor and the surface Green strain tensor, respectively. $\boldsymbol{q}_{s}^{0}$ is the surface heat flux vector, $q_{b}^{0}$ denotes the heat flow entering the surface from the bulk, $r_{s}^{0}$ is the surface heat supply, $T_{s}$ and $T_{b}$ are the temperatures of the surface and the bulk, $\varepsilon_{s}^{0}$ and $\eta_{s}^{0}$ denote respectively the surface internal energy and surface entropy per unit area, dot ( ) represents the rate of a quantity, and $\nabla_{0 s}$ is the surface gradient operators defined in the reference configuration. In the thermodynamic restrictions (2), "=" and " $<$ " hold for reversible and irreversible processes, respectively.

Introduce surface free energy per unit reference configuration area

$$
w_{s}^{0}=\varepsilon_{s}^{0}-T_{s} \eta_{s}^{0} .
$$

The surfaces energy balance and Clausius-Duhem inequality can be written as:

$$
\begin{aligned}
\left(\frac{\partial w_{s}^{0}}{\partial \boldsymbol{E}_{s}}-\boldsymbol{T}_{s}\right): \dot{\boldsymbol{E}}_{s}+\left(\frac{\partial w_{s}^{0}}{\partial T_{s}}+\eta_{s}^{0}\right) \dot{T}_{s} \\
\quad+T_{s} \dot{\eta}_{s}^{0}+\nabla_{0 s} \cdot \boldsymbol{q}_{s}^{0}-q_{b}^{0}-r_{s}^{0}=0
\end{aligned}
$$

and

$$
\left(\frac{\partial w_{s}^{0}}{\partial \boldsymbol{E}_{s}}-\boldsymbol{T}_{s}\right): \dot{\boldsymbol{E}}_{s}+\left(\frac{\partial w_{s}^{0}}{\partial T_{s}}+\eta_{s}^{0}\right) \dot{T}_{s}+R_{s}^{0} \leqslant 0
$$

where

$$
R_{s}^{0}=\boldsymbol{q}_{s}^{0} \cdot\left(\nabla_{0 s} T_{s}\right)+q_{b}^{0} \frac{T_{s}-T_{b}}{T_{b}} .
$$

Since $\dot{\boldsymbol{E}}_{s}$ and $\dot{T}_{s}$ are chosen arbitrarily, we have

$$
\begin{gathered}
\boldsymbol{T}_{s}=\frac{\partial w_{s}^{0}}{\partial \boldsymbol{E}_{s}}, \\
\eta_{s}^{0}=-\frac{\partial w_{s}^{0}}{\partial T_{s}}, \\
T_{s} \dot{\eta}_{s}^{0}+\nabla_{0 s} \cdot \boldsymbol{q}_{s}^{0}-q_{b}^{0}-r_{s}^{0}=0,
\end{gathered}
$$

and

$$
R_{s}^{0} \leqslant 0
$$

Eqs. (7)-(10) are the thermo-hyperelastic models of the surface. Correspondingly, we can define the surface specific heat at constant volume $C_{S E}$ as

$$
C_{s E}=\frac{\partial \varepsilon_{s}^{0}}{\partial T_{s}}=T_{s} \frac{\partial \eta_{s}^{0}}{\partial T_{s}}=-T_{s} \frac{\partial^{2} w_{s}^{0}}{\partial^{2} T_{s}} .
$$

Substituting eq. (8) into eq. (9), we get the surface heat conduction equation

$$
\nabla_{0 s} \cdot \boldsymbol{q}_{s}^{0}=T_{s} \boldsymbol{\alpha}_{s}: \dot{\boldsymbol{E}}_{s}-C_{s E} \dot{T}_{s}+q_{b}^{0}+r_{s}^{0},
$$

in which the second-order symmetric tensor $\boldsymbol{\alpha}_{s}=\frac{\partial w_{s}^{0}}{\partial T_{s} \partial \boldsymbol{E}_{s}}$ $=\frac{\partial \boldsymbol{T}_{s}}{\partial T_{s}}$ represents the surface thermal expansion properties.

Relations between the surface heat flux and the surface temperature can be modeled as in ref. [11]

$$
\boldsymbol{q}_{s}^{0}=\boldsymbol{q}_{s}^{0}\left(T_{s}, \boldsymbol{E}_{s}, \nabla_{0 s} T_{s}\right)
$$

which should satisfy the thermodynamic restriction (10).

Introduce the surface energy $\gamma$ per unit current configura- 
tion area, which is the function of the surface temperature $T_{s}$ and two invariants $\left(J_{1}\right.$ and $\left.J_{2}\right)$ of surface strain $\boldsymbol{E}_{s}$ for an isotropic solid surface. Hence, we obtain

$$
\boldsymbol{T}_{s}=\frac{\partial w_{s}^{0}\left(T_{s}, J_{1}, J_{2}\right)}{\partial \boldsymbol{E}_{s}}=\frac{\partial\left[J_{2} \gamma\left(T_{s}, J_{1}, J_{2}\right)\right]}{\partial \boldsymbol{E}_{s}}
$$

and

$$
\eta_{s}^{0}=-\frac{\partial\left[J_{2} \gamma\left(T_{s}, J_{1}, J_{2}\right)\right]}{\partial T_{s}} .
$$

Eq. (14) is similar to that given in ref. [18], but here we emphasize that the surface energy also depends on the temperature.

Surface heat flux can be expressed as

$$
\boldsymbol{q}_{s}^{0}=\left(k_{s}^{0} \boldsymbol{I}_{0}+k_{s}^{1} \boldsymbol{E}_{s}\right) \cdot \nabla_{0 s} T_{s},
$$

where $k_{s}^{0}$ and $k_{s}^{1}$ are the functions of the invariants of $\boldsymbol{E}_{s}$ and $\nabla_{0 s} T_{s}$, namely the functions of $J_{1}, J_{2}, T_{s}$, $\nabla_{0 s} T_{s} \cdot \nabla_{0 s} T_{s}$ and $\nabla_{0 s} T_{s} \cdot \boldsymbol{E}_{s} \cdot \nabla_{0 s} T_{s}$. Especially, if $q_{B}^{0}=$ 0 and $k_{s}^{0}=-k_{s}, k_{s}^{1}=0$, then eq. (16) can be simplified as the Fourier's law

$$
\boldsymbol{q}_{s}^{0}=-k_{s} \nabla_{0 s} T_{s}
$$

where $k_{s}$ is the isotropic surface heat conductivity.

\subsection{Infinitesimal surface thermoelasticity}

In the cases of infinitesimal deformations, surface strain $\boldsymbol{E}_{s}$ can be approximately expressed as $\boldsymbol{E}_{s}=\frac{1}{2}\left(\bar{\nabla}_{0 s} \boldsymbol{u}_{0}\right.$ $\left.+\boldsymbol{u}_{0} \bar{\nabla}_{0 s}\right)$ [21]. The invariants $J_{1}$ and $J_{2}$ of right surface stretch tensor $\boldsymbol{U}_{s}$ can be written as $J_{1}=2+\operatorname{tr} \boldsymbol{E}_{s}, J_{2}=1$ $+\operatorname{tr} \boldsymbol{E}_{s}+\operatorname{det} \boldsymbol{E}_{s} \quad[19,21]$. Note the change of the surface temperature $\theta_{s}=T_{s}-T_{s}^{0}$ is also not very large, where $T_{s}^{0}$ is the reference temperature. Hence, the temperature-related isotropic surface energy $\gamma$ may be expressed as the following series form

$$
\begin{aligned}
\gamma= & \underbrace{\gamma_{0}\left(T_{s}^{0}\right)}_{\text {initial surface energy }} \\
& +\underbrace{\gamma_{1}\left(J_{1}-2\right)+\gamma_{2}\left(J_{2}-1\right)+\frac{1}{2} \gamma_{11}\left(J_{1}-2\right)^{2}+\gamma_{12}\left(J_{1}-2\right)\left(J_{2}-1\right)+\frac{1}{2} \gamma_{22}\left(J_{2}-1\right)^{2}}_{\text {strain-contirbution surface energy [19,21] }} \\
& +\underbrace{\gamma_{3} \theta_{s}+\gamma_{31} \theta_{s}\left(J_{1}-2\right)+\gamma_{32} \theta_{s}\left(J_{2}-1\right)+\gamma_{33} \theta_{s}^{2}}_{\text {temperature-contribution surface energy }}+\ldots,
\end{aligned}
$$

where only second-order small quantities are considered. Substituting eq. (18) into eq. (14), and only keeping the first-order quantities, we get

$$
\begin{aligned}
\boldsymbol{T}_{s}=\gamma_{0}^{*} \boldsymbol{I}_{0} & +\left(\gamma_{0}^{*}+\gamma_{1}^{*}\right) \operatorname{tr}\left(\boldsymbol{E}_{s}\right) \boldsymbol{I}_{0} \\
& +\left(\gamma_{1}-2 \gamma_{0}^{*}\right) \boldsymbol{E}_{s}+\beta_{s} \theta_{s} \boldsymbol{I}_{0},
\end{aligned}
$$

where $\gamma_{0}^{*}=\gamma_{0}+\gamma_{1}+\gamma_{2}, \quad \gamma_{1}^{*}=\gamma_{1}+2 \gamma_{2}+\gamma_{11}+2 \gamma_{12}+\gamma_{22}$ $[19,21] ; \quad \beta_{s}=\gamma_{3}+\gamma_{31}+\gamma_{32}$ is the coefficients related to isotropic surface thermal expansion. Eq. (19) is the linear surface thermoelasticity described in the reference configuration.

The first kind surface Piola-Kirchhoff stress $\boldsymbol{S}_{s}=\boldsymbol{F}_{s} \cdot \boldsymbol{T}_{s}$ $[18,21]$, thus

$$
\begin{array}{r}
\boldsymbol{S}_{s}=\gamma_{0}^{*} \boldsymbol{I}_{0}+\left(\gamma_{0}^{*}+\gamma_{1}^{*}\right)\left(\operatorname{tr} \boldsymbol{E}_{s}\right) \boldsymbol{I}_{0}-\gamma_{0}^{*}\left(\bar{\nabla}_{0 s} \boldsymbol{u}_{0}\right) \\
+\gamma_{1} \boldsymbol{E}_{s}+\gamma_{0}^{*} \boldsymbol{F}_{s}^{(o)}+\beta_{s} \theta_{s} \boldsymbol{I}_{0} .
\end{array}
$$

The first kind Piola-Kirchhoff surface stress without temperature effects has been used by Huang and Sun to predict the effective moduli of nanocomposites [19].

Under Euler descriptions, surface Cauchy stress is de- fined as $\boldsymbol{\sigma}_{s}=\frac{1}{J_{2}} \boldsymbol{F}_{s} \cdot \boldsymbol{T}_{s} \cdot \boldsymbol{F}_{s}^{\mathrm{T}}[18,21]$. Under the basis of the reference configuration, the surface Cauchy stress can be represented as

$$
\begin{aligned}
\boldsymbol{\sigma}_{s}=\gamma_{0}^{*} \boldsymbol{I}_{0} & +\gamma_{1}^{*}\left(\operatorname{tr} \boldsymbol{E}_{s}\right) \boldsymbol{I}_{0}+\gamma_{1} \boldsymbol{E}_{s} \\
& +\gamma_{0}^{*}\left(\boldsymbol{F}_{s}^{(o)}+\boldsymbol{F}_{s}^{(o) T}\right)+\beta_{s} \theta_{s} \boldsymbol{I}_{0} .
\end{aligned}
$$

Eqs. (19), (20) and (21) imply that even in the cases of infinitesimal deformation, surface thermoelastic models described in different configurations are not the same due to the existence of surface tension $\gamma_{0}^{*}$.

When $\theta_{s}$ is not very large, the surface temperature in eq. (12) can be chosen as $T_{s}^{0}, \boldsymbol{\alpha}_{s}=\beta_{s} \boldsymbol{I}_{0}$, and the surface specific heat $C_{s E} \approx 2 T_{s}^{0} \gamma_{33}$. Hence, the surface heat conduction equation is reduced to

$$
C_{s E} \dot{T}_{s}=k_{s} T_{s, \alpha \alpha}+\beta_{s} T_{s} \operatorname{tr}\left(\dot{\boldsymbol{E}}_{s}\right)+q_{b}^{0}+r_{s}^{0} .
$$

The term $\beta_{s} T_{s} \operatorname{tr}\left(\dot{\boldsymbol{E}}_{s}\right)$ in eq. (22) implies that only the change of surface area can induce the couple phenomenon 
of the deformation and thermal effects. This couple behavior can be regarded as one kind of heat supply.

The aforementioned surface heat conduction equations are based on the Fourier's law and of parabolic type, in which the heat propagation speed is assumed to be infinite. However, if the heat propagation time is comparatively equivalent to the time of phonon mean free path, the finite speed needs to be considered. Correspondingly, the surface heat conduction equations should be modified.

\section{Thermoelasticity with residual stresses}

In the absence of external (mechanical or thermal) loadings, the residual surface stresses will induce an elastic field in the bulk materials. This self equilibrium state (without external loadings) is usually chosen as the reference configuration. That is to say, the bulk will deform from the residual stress states. Hence, the bulk of nanostructures will experience three configurations during deformations, namely the stress-free configuration, the reference configuration and the current configuration (as shown in Figure 1). The above three-configuration concept was first introduced by Huang in 2004 and discussed in detail in ref. [18], and the elasticity with residual stress for the bulk materials was discussed in ref. [21]. In the following we present the geometrical relations of different configuration descriptions.

\subsection{Geometrical relations}

Let $\boldsymbol{P}_{i}$ and $\boldsymbol{A}_{i}$ denote respectively the covariant base vectors of the stress-free configuration and the reference configuration, and assume that the temperatures of two configurations are the same, denoted by $T_{b}^{0}$. From the reference configuration to the current configuration, the displacement

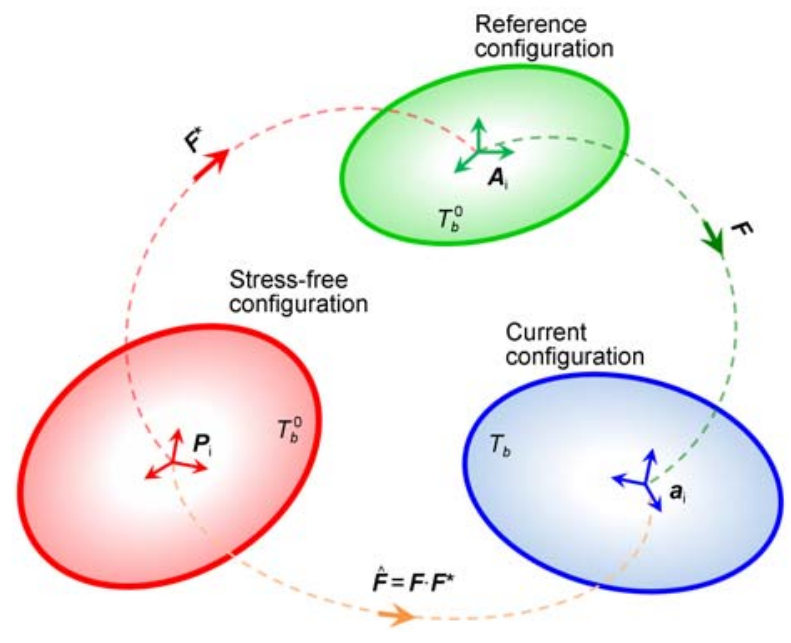

Figure 1 The choice of the reference configuration: the stressed state. of the bulk is $\boldsymbol{u}$, and the temperature change is $\theta_{b}=T_{b}$ $-T_{b}^{0}$. Therefore, the deformation gradients from the stressfree to the reference configurations, from the reference to the current configurations and from the stress-free to the current configurations are defined, respectively, as (see Figure 1)

$$
\begin{gathered}
\boldsymbol{F}^{*}=\boldsymbol{A}_{i} \otimes \boldsymbol{P}^{i}, \\
\boldsymbol{F}=\left(\boldsymbol{A}_{i}+\boldsymbol{u}_{, i}\right) \otimes \boldsymbol{A}^{i}=\boldsymbol{I}+\boldsymbol{u} \nabla,
\end{gathered}
$$

and

$$
\begin{aligned}
\hat{\boldsymbol{F}} & =\left(\boldsymbol{A}_{i}+\boldsymbol{u}_{, i}\right) \otimes \boldsymbol{P}^{i} \\
& =\left[\left(\boldsymbol{A}_{i}+\boldsymbol{u}_{, i}\right) \otimes \boldsymbol{A}^{i}\right] \cdot\left(\boldsymbol{A}_{i} \otimes \boldsymbol{P}^{i}\right)=\boldsymbol{F} \cdot \boldsymbol{F}^{*},
\end{aligned}
$$

where $\boldsymbol{P}^{i}$ and $\boldsymbol{A}^{i}$ are the contra-variant base vectors of $\boldsymbol{P}_{i}$ and $\boldsymbol{A}_{i}$, respectively. The symbol $(\hat{)}$ denotes the quantity defined in the stress-free configuration in this paper.

Correspondingly, the Green deformation tensors from the stress-free to the reference configurations, from the reference to the current configurations and from the stress-free to the current configurations are respectively

$$
\begin{gathered}
\boldsymbol{C}^{*}=\boldsymbol{F}^{* \mathrm{~T}} \cdot \boldsymbol{F}^{*}=\left(\boldsymbol{A}_{i} \cdot \boldsymbol{A}_{j}\right) \boldsymbol{P}^{i} \otimes \boldsymbol{P}^{j}, \\
\boldsymbol{C}=\boldsymbol{F}^{\mathrm{T}} \cdot \boldsymbol{F}=\boldsymbol{I}+2 E_{i j} \boldsymbol{A}^{i} \otimes \boldsymbol{A}^{j},
\end{gathered}
$$

and

$$
\hat{\boldsymbol{C}}=\hat{\boldsymbol{F}}^{\mathrm{T}} \cdot \hat{\boldsymbol{F}}=\boldsymbol{C}^{*}+2 E_{i j} \boldsymbol{P}^{i} \otimes \boldsymbol{P}^{j},
$$

where

$$
2 E_{i j}=\boldsymbol{u}_{, i} \cdot \boldsymbol{A}_{j}+\boldsymbol{A}_{i} \cdot \boldsymbol{u}_{, j}+\boldsymbol{u}_{, i} \cdot \boldsymbol{u}_{, j} .
$$

The corresponding strain tensors are defined as

$$
\begin{gathered}
\boldsymbol{E}^{*}=\frac{1}{2}\left(\boldsymbol{C}^{*}-\boldsymbol{I}\right), \\
\boldsymbol{E}=\frac{1}{2}(\boldsymbol{C}-\boldsymbol{I})=E_{i j} \boldsymbol{A}^{i} \otimes \boldsymbol{A}^{j},
\end{gathered}
$$

and

$$
\hat{\boldsymbol{E}}=\frac{1}{2}(\hat{\boldsymbol{C}}-\boldsymbol{1})=\boldsymbol{E}^{*}+\hat{\boldsymbol{E}}^{\prime}
$$

in which

$$
\hat{\boldsymbol{E}}^{\prime}=E_{i j} \boldsymbol{P}^{i} \otimes \boldsymbol{P}^{j}
$$

Assume the deformation is infinitesimal. Then

$$
E_{i j}=\frac{1}{2}\left(\boldsymbol{u}_{, i} \cdot \boldsymbol{A}_{j}+\boldsymbol{A}_{i} \cdot \boldsymbol{u}_{, j}\right) .
$$


from eqs. (31) and (33), it is found that $\boldsymbol{E}$ and $\hat{\boldsymbol{E}}^{\prime}$ are different strain tensors with the same components. Eq. (31) describes the strain defined in the reference configuration, but eq. (33) describes the change of the strain defined in the stress-free configuration, where $\boldsymbol{E}^{*}$ is the residual strain.

\subsection{Thermoelasticity with residual stresses}

\subsubsection{Expressions in the stress-free configuration}

Similar to the derivation of surface thermoelasticity, from the energy balance and Clausius-Duhem inequality [23-25], we can obtain the governing equations of the bulk in the stress-free configuration, as follows,

$$
\begin{aligned}
& \hat{\boldsymbol{T}}=\frac{\partial w\left(\hat{\boldsymbol{E}}, T_{b}\right)}{\partial \hat{\boldsymbol{E}}}, \\
& \hat{\eta}=-\frac{\partial w\left(\hat{\boldsymbol{E}}, T_{b}\right)}{\partial T_{b}}, \\
& T_{b} \dot{\hat{\eta}}+\hat{\nabla} \cdot \hat{\boldsymbol{q}}_{b}-\hat{r}_{b}=0,
\end{aligned}
$$

where $w$ is the strain energy function, $\hat{\boldsymbol{T}}$ and $\hat{\eta}$ denote respectively the second kind Piola-Kirchhoff stress and entropy, $\hat{\boldsymbol{q}}_{b}$ is the bulk heat flux, and $\hat{r}_{b}$ is the bulk heat supply.

For infinitesimal deformation cases, the displacement $\boldsymbol{u}$ and the temperature change $\theta_{b}$ are very small. Hence, eq. (35) can be expressed as the Taylor series form near the reference state $\left(\boldsymbol{E}^{*}, T_{b}^{0}\right)$, thus

$$
\begin{aligned}
& \hat{\boldsymbol{T}}\left(\hat{\boldsymbol{E}}, T_{b}\right)= \hat{\boldsymbol{T}}\left(\boldsymbol{E}^{*}+\hat{\boldsymbol{E}}^{\prime}, T_{b}^{0}+\theta_{b}\right) \\
&=\hat{\boldsymbol{T}}\left(\boldsymbol{E}^{*}, T_{b}^{0}\right)+\left.\frac{\partial \hat{\boldsymbol{T}}}{\partial \hat{\boldsymbol{E}}}\right|_{\hat{\boldsymbol{E}}=\boldsymbol{E}^{*}, T_{b}=T_{b}^{0}}: \hat{\boldsymbol{E}}^{\prime} \\
&+\left.\frac{\partial \hat{\boldsymbol{T}}}{\partial T_{b}}\right|_{\hat{\boldsymbol{E}}=\boldsymbol{E}^{*}, T_{b}=T_{b}^{0}} \theta_{b}+\ldots \\
&=\hat{\boldsymbol{T}}\left(\boldsymbol{E}^{*}, T_{b}^{0}\right)+\hat{L}\left(\boldsymbol{E}^{*}, T_{b}^{0}\right): \hat{\boldsymbol{E}}^{\prime} \\
& \quad-\hat{\boldsymbol{L}}_{1}\left(\boldsymbol{E}^{*}, T_{b}^{0}\right) \theta_{b}+\ldots
\end{aligned}
$$

and

$$
\begin{aligned}
\hat{\eta}\left(\hat{\boldsymbol{E}}, T_{b}\right)= & \hat{\eta}\left(\boldsymbol{E}^{*}+\hat{\boldsymbol{E}}^{\prime}, T_{b}^{0}+\theta_{b}\right) \\
= & \hat{\eta}\left(\boldsymbol{E}^{*}, T_{b}^{0}\right)+\left.\frac{\partial \hat{\eta}}{\partial \hat{\boldsymbol{E}}}\right|_{\hat{\boldsymbol{E}}=\boldsymbol{E}^{*}}: \hat{\boldsymbol{E}}^{\prime} \\
& +\left.\frac{\partial \hat{\eta}}{\partial T_{b}}\right|_{T_{b}=T_{b}^{0}} \theta_{b}+\ldots \\
= & \hat{\eta}\left(\boldsymbol{E}^{*}, T_{b}^{0}\right)+\hat{\boldsymbol{L}}_{1}\left(\boldsymbol{E}^{*}, T_{b}^{0}\right): \hat{\boldsymbol{E}}^{\prime}
\end{aligned}
$$

$$
+\frac{\hat{C}_{E}\left(\boldsymbol{E}^{*}, T_{b}^{0}\right)}{T_{b}} \theta_{b}+\ldots
$$

in which the fourth-order tensor $\hat{L}=\frac{\partial^{2} w}{\partial \hat{\boldsymbol{E}} \partial \hat{\boldsymbol{E}}}$ is the isothermal elasticity tensor, the second-order tensor $\hat{\boldsymbol{L}}_{1}=-\frac{\partial^{2} w}{\partial T_{b} \partial \hat{\boldsymbol{E}}}$ is the stress-temperature modulus [25], $\hat{C}_{E}=-T_{b} \frac{\partial^{2} w}{\partial^{2} T_{b}}$ is the bulk specific heat at constant volume. In the same way, the heat flux can be represented as

$$
\begin{aligned}
\hat{\boldsymbol{q}}_{b}\left(\hat{\boldsymbol{E}}, T_{b}, \hat{\nabla} T_{b}\right)= & \hat{\boldsymbol{q}}_{b}\left(\boldsymbol{E}^{*}, T_{b}^{0}, \hat{\nabla} T_{b}^{0}\right) \\
& +\hat{\boldsymbol{L}}_{2}\left(\boldsymbol{E}^{*}, T_{b}^{0}, \hat{\nabla} T_{b}^{0}\right): \hat{\boldsymbol{E}}^{\prime} \\
& +\hat{\boldsymbol{L}}_{3}\left(\boldsymbol{E}^{*}, T_{b}^{0}, \hat{\nabla} T_{b}^{0}\right) \theta_{b} \\
& +\hat{\boldsymbol{L}}_{4}\left(\boldsymbol{E}^{*}, T_{b}^{0}, \hat{\nabla} T_{b}^{0}\right) \cdot \hat{\nabla} \theta_{b}+\ldots,
\end{aligned}
$$

where

$$
\begin{aligned}
& \hat{\boldsymbol{L}}_{2}\left(\boldsymbol{E}^{*}, T_{b}^{0}, \hat{\nabla} T_{b}^{0}\right)=\left.\frac{\partial \hat{\boldsymbol{q}}_{b}}{\partial \hat{\boldsymbol{E}}}\right|_{\hat{\boldsymbol{E}}=\boldsymbol{E}^{*}, T_{b}=T_{b}^{0}, \hat{\nabla} T_{b}=\hat{\nabla} T_{b}^{0}}, \\
& \hat{\boldsymbol{L}}_{3}\left(\boldsymbol{E}^{*}, T_{b}^{0}, \hat{\nabla} T_{b}^{0}\right)=\left.\frac{\partial \hat{\boldsymbol{q}}_{b}}{\partial T_{b}}\right|_{\hat{\boldsymbol{E}}=\boldsymbol{E}^{*}, T_{b}=T_{b}^{0}, \hat{\nabla} T_{b}=\hat{\nabla} T_{b}^{0}}, \\
& \hat{\boldsymbol{L}}_{4}\left(\boldsymbol{E}^{*}, T_{b}^{0}, \hat{\nabla} T_{b}^{0}\right)=\left.\frac{\partial \hat{\boldsymbol{q}}_{b}}{\partial\left(\hat{\nabla} T_{b}\right)}\right|_{\hat{\boldsymbol{E}}=\boldsymbol{E}^{*}, T_{b}=T_{b}^{0}, \hat{\nabla} T_{b}=\hat{\nabla} T_{b}^{0}} .
\end{aligned}
$$

\subsubsection{Expressions in the reference configuration}

The aforementioned equations are expressed in the stressfree configuration. However, the given configuration is usually the reference or the current one. Now, we will present the expressions of these governing equations in other configurations. Firstly, the first kind Piola-Kirchhoff stress $S$, defined from the reference configuration to the current one, is [18]

$$
\boldsymbol{S}=\frac{1}{J^{*}} \hat{\boldsymbol{F}} \cdot \hat{\boldsymbol{T}} \cdot \boldsymbol{F}^{* \mathrm{~T}}=\frac{1}{J^{*}} \boldsymbol{F} \cdot \boldsymbol{F}^{*} \cdot \hat{\boldsymbol{T}} \cdot \boldsymbol{F}^{* \mathrm{~T}},
$$

in which $J^{*}=\operatorname{det}\left(\boldsymbol{F}^{*}\right)$. Substituting eq. (36) into the above equation and keeping the first-order terms of deformation gradient, we get

$$
\begin{aligned}
\boldsymbol{S}=\boldsymbol{T}_{R}+\boldsymbol{u} \nabla \cdot \boldsymbol{T}_{R} & +\frac{1}{J^{*}} \boldsymbol{F}^{*} \cdot\left[\hat{L}\left(\boldsymbol{E}^{*}, T_{b}^{0}\right): \hat{\boldsymbol{E}}^{\prime}\right] \cdot \boldsymbol{F}^{* \mathrm{~T}} \\
& -\frac{1}{J^{*}} \boldsymbol{F}^{*} \cdot \hat{\boldsymbol{L}}_{1}\left(\boldsymbol{E}^{*}, T_{b}^{0}\right) \cdot \boldsymbol{F}^{* \mathrm{~T}} \theta_{b},
\end{aligned}
$$

where $\boldsymbol{T}_{R}=\frac{1}{J^{*}} \boldsymbol{F}^{*} \cdot \hat{\boldsymbol{T}}\left(\boldsymbol{E}^{*}, T_{b}^{0}\right) \cdot \boldsymbol{F}^{* \mathrm{~T}}$ denotes the residual 
stress measured in the reference configuration. Note that

$$
\begin{aligned}
& \hat{L}\left(\boldsymbol{E}^{*}, T_{b}^{0}\right): \hat{\boldsymbol{E}}^{\prime} \\
& \quad=\left(\frac{\partial^{2} w}{\partial E_{i j}^{*} \partial E_{k l}^{*}} \boldsymbol{P}_{i} \otimes \boldsymbol{P}_{j} \otimes \boldsymbol{P}_{k} \otimes \boldsymbol{P}_{l}\right):\left(E_{m n} \boldsymbol{P}^{m} \otimes \boldsymbol{P}^{n}\right) \\
& \quad=\frac{\partial^{2} w}{\partial E_{i j}^{*} \partial E_{k l}^{*}} E_{k l} \boldsymbol{P}_{i} \otimes \boldsymbol{P}_{j}, \\
& \hat{\boldsymbol{L}}_{1}\left(\boldsymbol{E}^{*}, T_{b}^{0}\right)=-\frac{\partial^{2} w}{\partial T_{b} \partial E_{i j}^{*}} \boldsymbol{P}_{i} \otimes \boldsymbol{P}_{j},
\end{aligned}
$$

where $E_{i j}^{*}$ are the covariant components of $\boldsymbol{E}^{*}$ defined in the stress-free configuration. Thus, the terms in eq. (41) can be written as

$$
\begin{aligned}
& \frac{1}{J^{*}} \boldsymbol{F}^{*} \cdot\left[\hat{L}\left(\boldsymbol{E}^{*}, T_{b}^{0}\right): \hat{\boldsymbol{E}}^{\prime}\right] \cdot \boldsymbol{F}^{* \mathrm{~T}} \\
& \quad=\frac{1}{J^{*}} \frac{\partial^{2} w}{\partial E_{i j}^{*} \partial E_{k l}^{*}} E_{k l} \boldsymbol{A}_{i} \otimes \boldsymbol{A}_{j}=L\left(\boldsymbol{E}^{*}, T_{b}^{0}\right): \boldsymbol{E}, \\
& \frac{1}{J^{*}} \boldsymbol{F}^{*} \cdot \hat{\boldsymbol{L}}_{1}\left(\boldsymbol{E}^{*}, T_{b}^{0}\right) \cdot \boldsymbol{F}^{* \mathrm{~T}} \\
& =-\frac{1}{J^{*}} \frac{\partial^{2} w}{\partial T_{b} \partial E_{i j}^{*}} \boldsymbol{A}_{i} \otimes \boldsymbol{A}_{j}=\boldsymbol{L}_{1}\left(\boldsymbol{E}^{*}, T_{b}^{0}\right),
\end{aligned}
$$

where

$$
L\left(\boldsymbol{E}^{*}, T_{b}^{0}\right)=\left(\frac{1}{J^{*}} \frac{\partial^{2} w}{\partial E_{i j}^{*} \partial E_{k l}^{*}} \boldsymbol{A}_{i} \otimes \boldsymbol{A}_{j} \otimes \boldsymbol{A}_{k} \otimes \boldsymbol{A}_{l}\right)
$$

is the isothermal elasticity tensor defined in the reference configuration, $\boldsymbol{L}_{1}\left(\boldsymbol{E}^{*}, T_{b}^{0}\right)$ is the stress-temperature modulus.

Thus

$$
\boldsymbol{S}=\boldsymbol{T}_{R}+\boldsymbol{u} \nabla \cdot \boldsymbol{T}_{R}+L\left(\boldsymbol{E}^{*}, T_{b}^{0}\right): \boldsymbol{E}-\boldsymbol{L}_{1}\left(\boldsymbol{E}^{*}, T_{b}^{0}\right) \theta_{b} .
$$

The entropy per unit volume in the reference configuration, $\eta=\frac{1}{J^{*}} \hat{\eta}$, should satisfy

$$
\eta=\eta_{R}+\boldsymbol{L}_{1}\left(\boldsymbol{E}^{*}, T_{b}^{0}\right): \boldsymbol{E}+\frac{C_{E}\left(\boldsymbol{E}^{*}, T_{b}^{0}\right)}{T_{b}} \theta_{b},
$$

in which $\eta_{R}=\frac{1}{J^{*}} \hat{\eta}\left(\boldsymbol{E}^{*}, T_{b}^{0}\right)$ and $C_{E}=\frac{1}{J^{*}} \hat{C}_{E}$ are the residual entropy and specific heat at a constant volume in the reference configuration, respectively.

Next, the heat conduction equation will also be presented in the reference configuration. Define heat flux $\boldsymbol{q}_{b}^{0}=$ $\frac{1}{J^{*}} \boldsymbol{F}^{*} \cdot \hat{\boldsymbol{q}}_{b}$ in the reference configuration, thus

$$
\begin{aligned}
\boldsymbol{q}_{b}^{0} & =\frac{1}{J^{*}} \boldsymbol{F}^{*} \cdot \hat{\boldsymbol{q}}_{b}\left(\hat{\boldsymbol{E}}, T_{b}, \hat{\nabla} T_{b}\right) \\
& =\hat{\boldsymbol{q}}_{b}^{R}+\boldsymbol{L}_{2}: \boldsymbol{E}+\boldsymbol{L}_{3} \theta_{b}+\boldsymbol{L}_{4} \cdot \nabla_{0} \theta_{b},
\end{aligned}
$$

where $\hat{\boldsymbol{q}}_{b}^{R}=\frac{1}{J^{*}} \boldsymbol{F}^{*} \cdot \hat{\boldsymbol{q}}_{b}\left(\boldsymbol{E}^{*}, T_{b}^{0}, \hat{\nabla} T_{b}^{0}\right)$ is the initial value of $\boldsymbol{q}_{b}^{0} . \boldsymbol{L}_{2}, \boldsymbol{L}_{3}$ and $\boldsymbol{L}_{4}$ are defined as

$$
\begin{aligned}
& \boldsymbol{L}_{2}=\frac{1}{J^{*}} \boldsymbol{F}^{*} \cdot \frac{\partial \hat{\boldsymbol{q}}_{b}}{\partial E_{i j}^{*}} \boldsymbol{A}_{i} \otimes \boldsymbol{A}_{j}, \\
& \boldsymbol{L}_{3}=\frac{1}{J^{*}} \boldsymbol{F}^{*} \cdot \hat{\boldsymbol{L}}_{3}, \\
& \boldsymbol{L}_{4}=\frac{1}{J^{*}} \boldsymbol{F}^{*} \cdot \frac{\partial \hat{\boldsymbol{q}}_{b}}{\partial\left(T_{b, i}\right)} \boldsymbol{A}_{i} .
\end{aligned}
$$

whereby, the heat conduction equation can be written as

$$
T_{b} \dot{\eta}+\nabla_{0} \cdot \boldsymbol{q}_{b}^{0}-r_{b}^{0}=0 .
$$

The Cauchy stress $\sigma$ may be expressed as

$$
\begin{aligned}
\boldsymbol{\sigma}= & \frac{1}{J} \boldsymbol{S} \cdot \boldsymbol{F}^{\mathrm{T}} \\
= & {[1-\operatorname{tr}(\boldsymbol{E})] \boldsymbol{T}_{R}+\boldsymbol{u} \nabla \cdot \boldsymbol{T}_{R}+\boldsymbol{T}_{R} \cdot \nabla \boldsymbol{u} } \\
& +L\left(\boldsymbol{E}^{*}, T_{b}^{0}\right): \boldsymbol{E}-\boldsymbol{L}_{1}\left(\boldsymbol{E}^{*}, T_{b}^{0}\right) \theta_{b},
\end{aligned}
$$

where $J=\operatorname{det}(\boldsymbol{F}) \approx 1-\operatorname{tr}(\boldsymbol{E})$.

\subsection{Linear thermoelasticity with residual stresses}

In this subsection, the linear isotropic thermoelasticity with residual stresses will be considered. Assume that [23]

$$
L\left(\boldsymbol{E}^{*}, T_{b}^{0}\right)=\lambda \boldsymbol{I} \otimes \mathbf{1}+2 \mu \mathbf{1} \otimes \boldsymbol{I}
$$

and

$$
\boldsymbol{L}_{1}\left(\boldsymbol{E}^{*}, T_{b}^{0}\right)=-\beta_{b} \mathbf{1}
$$

where $\lambda$ and $\mu$ are the bulk Lamé constants, $\beta_{b}$ is the bulk thermal expansion coefficient, the fourth-order tensors $1 \otimes 1$ and $1 \otimes 1$ are defined as $(1 \otimes I): A=\operatorname{tr}(A) \boldsymbol{I}$ and $(\mathbf{1} \mathbb{1}): \boldsymbol{A}=\boldsymbol{A}$, respectively, in which $\boldsymbol{A}$ is a second-order tensor [26]. Substituting eqs. (51) and (52) into eqs. (45) and (46), we have

$$
\boldsymbol{S}=\boldsymbol{T}_{R}+\boldsymbol{u} \nabla \cdot \boldsymbol{T}_{R}+\lambda \operatorname{tr}(\boldsymbol{E}) \boldsymbol{I}+2 \mu \boldsymbol{E}+\beta_{b} \theta_{b} \boldsymbol{I}
$$

and

$$
\eta=\eta_{R}-\beta_{b} \operatorname{tr}(\boldsymbol{E})+\frac{C_{E} \theta_{b}}{T_{b}^{0}} .
$$


For isotropic materials, $\boldsymbol{L}_{2}=0, \boldsymbol{L}_{3}=0$ and $\boldsymbol{L}_{4}=-k_{b} \boldsymbol{1}$. Thus, the Fourier's law is

$$
\boldsymbol{q}_{b}^{0}=\hat{\boldsymbol{q}}_{b}^{R}-k_{b} \nabla_{0} \theta_{b} .
$$

Assume that the reference configuration is in equilibrium state. Then, $\nabla_{0} \cdot \hat{\boldsymbol{q}}_{b}^{R}=0$ and $\dot{\eta}_{R}=0$. Substituting eqs. (54) and (55) into eq. (49), the heat conduction equation is reduced to

$$
C_{E} \dot{\theta}_{b}=\beta_{b} T_{b}^{0} \operatorname{tr}(\dot{\boldsymbol{E}})+k_{b} \nabla_{0}^{2} \theta_{b}+r_{b}^{0} .
$$

\section{Thermoelastic expansion of nanoparticles}

Nanomaterials exhibit many new properties different from traditional macroscopic materials, such as surface and size effects, etc. In this section, the proposed equations are used to analyze the size effect of the effective thermal expansion coefficient of nanoparticles [27,28].

Consider an isotropic spherical nanoparticle, which is in the self equilibrium state without external loadings. The radius is $r_{0}$, and the temperature of the surface and the bulk is $T^{0}$. Figure 2 shows the structure of the nanosphere and the corresponding parameters. The stress field in the bulk induced by the surface residual stress will be studied in the following.

\subsection{Residual stresses in bulk}

Ignore the change of surface stresses in the self-equilibrium process, and let the surface residual stress be $\gamma_{0}^{*}$. Thus, the generalized Young-Laplace equation implies that

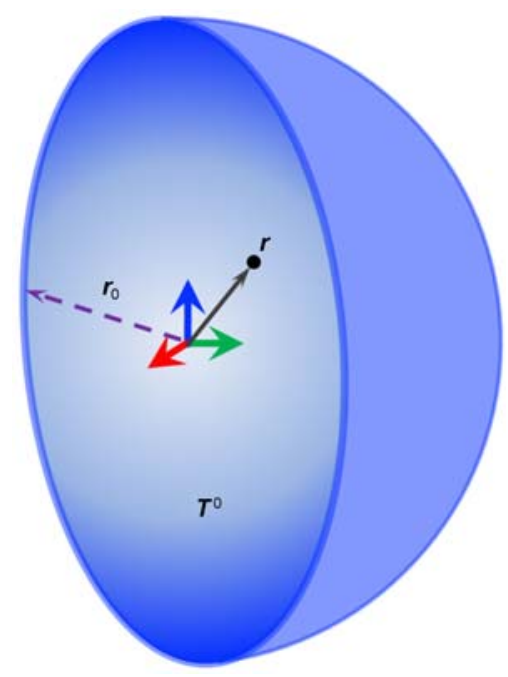

Figure 2 Structure for the isotropic spherical nanoparticle.

$$
\left.\hat{T}_{r r}\right|_{r=r_{0}}=-\frac{2 \gamma_{0}^{*}}{r_{0}} .
$$

Assume that in the stress-free configuration, the radius of this nanoparticle is $\bar{r}$. Due to spherical symmetry, the displacements under the action of surface residual stress can be expressed as

$$
\boldsymbol{u}^{*}(\boldsymbol{r})=f(r) \boldsymbol{r}
$$

where $r$ is the location vector, and $r=\|r\|$. It follows that

$$
\boldsymbol{E}^{*}=f(r) \boldsymbol{I}+\frac{1}{r} \frac{\mathrm{d} f(r)}{\mathrm{d} r} \boldsymbol{r} \otimes \boldsymbol{r} .
$$

Substituting eq. (59) into classical Hooke's law and equilibrium equation, thus

$$
(\lambda+2 \mu)\left(4 \frac{\mathrm{d} f}{\mathrm{~d} r}+r \frac{\mathrm{d}^{2} f}{\mathrm{~d} r^{2}}\right)=0 .
$$

Since $f(0)=0$, we get $f(r)=\bar{\alpha}$, where $\bar{\alpha}$ is a constant. Therefore, the residual stress in the bulk is

$$
\hat{T}_{r r}=\bar{\alpha}(3 \lambda+2 \mu)
$$

Replacing $\hat{T}_{r r}$ in eq. (57) with eq. (61), and considering $r_{0}=(1+\bar{\alpha}) \bar{r}$, we obtain

$$
\bar{\alpha}=\frac{-1+\sqrt{1-\frac{8 \gamma_{0}^{*}}{\bar{r}(3 \lambda+2 \mu)}}}{2} .
$$

When $\bar{r}$ is very large or $\gamma_{0}^{*}$ is very small, $\bar{\alpha} \approx$ $-\frac{2 \gamma_{0}^{*}}{\bar{r}(3 \lambda+2 \mu)}$.

\subsection{Effective thermal expansion of nanoparticles}

If the temperature field is changed, the nanoparticles will expand or contract. The prestressed self equilibrium state without temperature change is chosen as the reference configuration. The displacement due to temperature change $\theta$ can be assumed as $\boldsymbol{u}=\alpha \boldsymbol{r}$, where $\alpha$ is a constant. The corresponding strain tensors are

$$
\boldsymbol{E}=\nabla \boldsymbol{u}=\alpha \boldsymbol{1}, \quad \boldsymbol{E}_{s}=\nabla_{s} \boldsymbol{u}=\alpha \boldsymbol{I}_{0} .
$$

Substituting eq. (63) into constitutive eqs. (20) and (53), and using the generalized Young-Laplace equations, we have

$$
\begin{aligned}
& -\left[\gamma_{0}^{*}+\alpha\left(\gamma_{0}^{*}+2 \gamma_{1}^{*}+\gamma_{1}\right)+\beta_{s} \theta\right] \frac{2}{r_{0}} \\
& =\bar{\alpha}(3 \lambda+2 \mu)(1+\alpha)+(3 \lambda+2 \mu) \alpha+\beta_{b} \theta .
\end{aligned}
$$


Thus

$$
\alpha=-\frac{\beta_{b}+\frac{2 \beta_{s}}{\bar{r}(1+\bar{\alpha})}}{\frac{2\left(\gamma_{0}^{*}+2 \gamma_{1}^{*}+\gamma_{1}\right)}{\bar{r}(1+\bar{\alpha})}+(1+\bar{\alpha})(3 \lambda+2 \mu)} \theta .
$$

Then, the effective thermal expansion coefficient of nanoparticles is

$$
\alpha_{e}=-\frac{\beta_{b}+\frac{2 \beta_{s}}{\bar{r}(1+\bar{\alpha})}}{\frac{2\left(\gamma_{0}^{*}+2 \gamma_{1}^{*}+\gamma_{1}\right)}{\bar{r}(1+\bar{\alpha})}+(1+\bar{\alpha})(3 \lambda+2 \mu)} .
$$

When surface residual stress $\gamma_{0}^{*}$ is not very large, eq. (66) is reduced to [29]

$$
\alpha_{e}=-\frac{\beta_{b}+\frac{2 \beta_{s}}{\bar{r}}}{\frac{2\left(\gamma_{0}^{*}+2 \gamma_{1}^{*}+\gamma_{1}\right)}{\bar{r}}+(3 \lambda+2 \mu)} .
$$

If the radius is large enough, the present result is reduced to the classical thermoelastic case [25]:

$$
\alpha_{e}=-\frac{\beta_{b}}{3 \lambda+2 \mu} \text {. }
$$

\section{Conclusions}

In the absence of external mechanical or thermal loadings, surface residual stresses will induce a stress field in the bulk of nanostructured materials. Under the framework of thermodynamics, the present study establishes the thermo-hyperelastic constitutive relations of the surface and the bulk of these materials, respectively. The residual stresses are taken into account. It is shown that even in the cases of infinitesimal deformation, different descriptions of the surface or the bulk constitutive relations should be discriminated due to the existence of the residual stresses. Thus, in the thermomechanical analyses of nanostructures, we should choose the appropriate constitutive equations. Furthermore, the example of effective thermal expansion coefficient of nanoparticles indicates that if the surface residual stress is large or the characteristic length is very small, the residual stresses should not be neglected.

This research was supported by the National Natural Science Foundation of China (Grant Nos. 60936001, 10772180, 10902111), the National Basic Research Program of China (Grant No. 2007CB310500), and the Foundamental Research Funds for the Central Universities (Grant No. 2010ZY33).
1 Hill T L. Thermodynamics of Small Systems. New York: Dover Pub. INC, 2002

2 Guo Z Y. Frontier of heat transfer-microscale heat transfer (in Chinese). Adv Mech, 2000, 30: 1-6

3 Cimalla V, Niebelschutz F, Tonisch K, et al. Nanoelectromechanical devices for sensing applications. Sensor Actuat B, 2007, 126: 24-34

4 Cleland A N. Foundations of Nanomechanics. Berlin: Springer, 2003

5 Gleiter H. Nanostructured materials: Basic concepts and microstructure. Acta Mater, 2000, 48: 1-29

6 Bhushan B. Handbook of Nanotechnology. 3rd Ed. Berlin: Springer, 2010

7 Tang Z, Zhao H, Li G, et al. Finite-temperature quasicontinuum method for multiscale analysis of silicon nanostructures. Phys Rev B, 2006, 74: 064110

8 Xiao S P, Yang W X. Temperature-related Cauchy-Born rule for multiscale modeling of crystalline solids. Comp Mater Sci, 2006, 37 : 374-379

9 Yang W X, Xiao S P. Extension of the temperature-related CauchyBorn rule: Material stability analysis and thermo-mechanical coupling. Comp Mater Sci, 2008, 41: 431-439

10 Yun G, Park H S. A multiscale, finite deformation formulation for surface stress effects on the coupled thermomechanical behavior of nanomaterials. Comput Method Appl Mech Eng, 2008, 197: 33373350

11 Murdoch A I. A thermodynamical theory of elastic material interfaces. Q J Mech Appl Math, 1976, 29: 245-275

12 Rusanov A. Thermodynamics of solid surfaces. Surf Sci Rep, 1996, 23: 173-247

13 Javili A, Steinmann P. On thermomechanical solids with boundary structures. Int J Solids Struct, 2010, 47: 3245-3253

14 Chen T, Dvorak G. Fibrous nanocomposites with interface stress: Hill's and Levin's connections for effective moduli. Appl Phys Lett, 2006, 88: 211912

15 Duan H L, Karihaloo B L. Thermo-elastic properties of heterogeneous materials with imperfect interfaces: Generalized Levin's formula and Hill's connections. J Mech Phys Solids, 2007, 55: 1036-1052

16 Gordeliy E, Mogilevskaya S G, Crouch S L. Transient thermal stresses in a medium with a circular cavity with surface effects. Int $\mathrm{J}$ Solids Struct, 2009, 46: 1834-1848

$17 \mathrm{Ru}$ C Q. Size effect of dissipative surface stress on quality factor of microbeams. Appl Phys Lett, 2009, 94: 051905

18 Huang Z P, Wang J. A theory of hyperelasticity of multi-phase media with surface/interface energy effect. Acta Mech, 2006, 182: 195-210

19 Huang Z P, Sun L. Size-dependent effective properties of a het-erogeneous material with interface energy effect: From finite defor-mation theory to infinitesimal strain analysis. Acta Mech, 2007, 190: $151-163$

20 Wang Z Q, Zhao Y P. Self-instability and bending behaviors of nano plates. Acta Mech Solida Sinica, 2009, 22: 630-643

21 Wang Z Q, Zhao Y P, Huang Z P. The effects of surface tension on the elastic properties of nano structures. Int J Eng Sci, 2010, 48: $140-150$

22 Ru C Q. Simple geometrical explanation of Gurtin-Murdoch model of surface elasticity with clarification of its related versions. Sci China Phys Mech Astron, 2010, 53: 536-544

23 Truesdell C, Noll W. The Non-linear Field Theories of Mechanics. Berlin: Springer, 2004

24 Hwang K C, Huang Y G. Solid Constitutive Relations (in Chinese). Beijing: Tsinghua University Press, 1999

25 Gurtin M E, Fried E, Anand L. The Mechanics and Thermodynamics of Continua. Cambridge: Cambridge University Press, 2009

26 Dui G S, Wang Z D, Jin M. Derivatives on the isotropic tensor functions. Sci China Ser G-Phys Mech Astron, 2006, 49: 321-334

27 Pathak S, Shenoy V B. Size dependence of thermal expansion of nanostructures. Phys Rev B, 2005, 72: 113404

28 Zhou L J, Guo J G, Zhao Y P. Size and temperature-dependent thermal expansion coefficient of a nanofilm. Chin Phys Lett, 2009, 26: 06620

29 Murdoch A I. Some fundamental aspects of surface modeling. J Elast, 2005, 80: 33-52 